\title{
GLAD!
}

Revue sur le langage, le genre, les sexualités

$01 \mid 2016$

Varia

\section{Inventer son genre dans le langage de la télévision}

Inventing One's Own Gender on Television

\section{Catherine Gonnard et Elisabeth Lebovici}

\section{(2) OpenEdition}

Journals

Édition électronique

URL : http://journals.openedition.org/glad/209

ISSN : 2551-0819

Éditeur

Association GSL

Référence électronique

Catherine Gonnard et Elisabeth Lebovici, «Inventer son genre dans le langage de la télévision », GLAD! [En ligne], 01 | 2016, mis en ligne le 15 novembre 2016, consulté le 17 décembre 2020. URL : http:// journals.openedition.org/glad/209

Ce document a été généré automatiquement le 17 décembre 2020.

\section{c) (i) $\Theta$}

La revue GLAD! est mise à disposition selon les termes de la Licence Creative Commons Attribution Pas d'Utilisation Commerciale - Pas de Modification 4.0 International. 


\title{
Inventer son genre dans le langage de la télévision
}

\author{
Inventing One's Own Gender on Television
}

Catherine Gonnard et Elisabeth Lebovici

1 Les histoires de la télévision française accordent peu de place aux émissions de divertissement dit «populaire » que sont les programmes de variétés des années 1950 et 1960. On y décrit les émissions de variétés d'après-guerre comme une émanation de la radio et du music-hall ${ }^{1}$, ou comme un genre spécifique de la télévision fortement influencé par l'audience et les pressions de l'industrie du disque ${ }^{2}$. Pour leur reconnaissance, il faut attendre les plateaux à invités multiples du couple Maritie et Gilbert Carpentier, à partir de 1961, ou les émissions de Jean-Christophe Averty, qui bousculent les téléspectateurs à partir de $1963^{3}$. Cependant, les questions de genre n'y sont guère abordées et sont même occultées quand il s'agit du couple Carpentier. Or, à la lecture des génériques, on découvre que les femmes y ont une place plus importante qu'on pourrait le croire et qu'elles y sont créditées du titre de productrices, de présentatrices et plus rarement de réalisatrices. D'un autre côté, les études qui s'attachent à décrire les représentations de l'homosexualité dans le divertissement télévisuel s'accrochent au "personnage » gay, lesbienne ou trans affiché.e comme tel.le, pour constater son absence au moins jusqu'au milieu des années 1970, et plus souvent jusqu'aux années $1990^{4}$. Le manque d'espace médiatique accordé aux communautés LGBT est, encore aujourd'hui, notable ${ }^{5}$.

2 Visionnant diverses émissions de variétés et lisant les magazines de programmes de la période de 1955 à $1969^{6}$, nous avons été intriguées par ce moment de l'histoire télévisuelle française, où les productrices des émissions de variétés ont été nombreuses, telles Aimée Mortimer, Mireille, Michèle Arnaud, Jacqueline Joubert, Denise Glaser ou Maritie Carpentier. Plus particulièrement, l'émission Cabaret $d u$ soir ${ }^{7}$, conférant un double statut de « productrices-présentatrices » à Colette Mars et Micheline Sandrel, a arrêté notre attention. Chanteuse, actrice, Colette Mars a aussi été la propriétaire du cabaret $L a$ Vie parisienne $e^{8}$, qu'elle a racheté à Suzy Solidor en 1947. Cette même année, Micheline Sandrel, journaliste, a fait ses premières armes à la radio et a commencé à 
collaborer dès 1954 au Journal télévisé puis au Magazine féminin. Associées à la fois à la production et à la présentation de leur émission, les deux femmes ont ainsi pu décider qui elles invitaient et comment elles allaient les rendre visibles, c'est-à-dire de quelles façons ces invité.e.s allaient " passer ${ }^{10}$ " à la télévision. Notre enquête a ainsi débuté par cette émission spécifiquement, puis s'est élargie à d'autres comme La Joie de vivre ${ }^{11}, 36$ chandelles, École des vedettes ${ }^{12}$, pour confirmer ou compléter nos informations sur certaines invitées de Colette Mars et Micheline Sandrel. Il nous est apparu rapidement que la double fonction de productrice et présentatrice allait de pair avec la présence réitérée d'《artistes ", chanteuses, actrices, performeuses, peintres, poètes, voire de figurant.e.s en arrière-plan. Cette visibilité que les productrices-présentatrices programmaient de façon répétitive à l'écran ne concernait pas seulement la "performance d'artiste» des invité.e.s. Nous avons constaté que Colette Mars et Micheline Sandrel allaient bien au-delà de la présentation du «numéro » (la chanson, le poème, le sketch de music-hall) et tenaient conversation avec leurs invité.e.s au cours de l'émission. Ces conversations, au-delà du discours promotionnel, faisaient état de façon manifeste ou plus latente de liens entre elles. Ainsi nous en sommes venues à nous demander quelle était la place - puisqu'il y en avait une - pour les sociabilités féminines dans l'espace télévisuel et comment on pouvait les identifier. Connaissant les connexions de Colette Mars avec le monde lesbien, notamment avec Suzy Solidor ${ }^{13}$, certains traits récurrents dans les émissions nous sont apparus comme des indices de ces relations, conduisant à imaginer l'ébauche d'un réseau. Nous nous sommes aperçu que la télévision et ses à-côtés - ses parerga que sont les journaux de programmes télévisés, les partitions de chansons, les couvertures de disques - proposaient un espace visuel et sonore où reconnaître ce qu'on pourrait aujourd'hui appeler une culture homosociale, perceptible même de façon diffuse à qui la recherche. Ainsi a-t-on pu formuler l'hypothèse que, loin des mouvements féministes et/ou lesbiens qui se sont développés à partir des années 1970, les expériences télévisuelles de Cabaret du soir, d'École des vedettes, de 36 chandelles puis de Discorama ${ }^{14}$ esquissent une réflexion sur des genres qui ne «collent» pas avec l'assignation de la féminité aux femmes, ni de la masculinité aux hommes, des années 1950-1970 en France.

3 Associer l'orientation sexuelle et l'expression de genre mérite ici une explication. Nous utilisons le terme de "genre(s)» avec la visée d'intégrer notre travail dans un champ d'études ${ }^{15}$ qui cartographie les façons de parler, les comportements, les attitudes et les gestes par lesquels l'individu.e se conforme et/ou brouille la structure binaire du modèle « femme »/ « homme » telle qu'elle est construite par une société. Or, le concept de genre s'élabore dans les années 1950, la période où les programmes télévisés que nous étudions sont produits et diffusés ${ }^{16}$. Ces années, qui précèdent celles où se constituent dans les sociétés occidentales des «identités homosexuelles ${ }^{17}$ " puis " gay et lesbiennes ${ }^{18} "$, sont une période de non-dit, durant laquelle ce qu'on appelle aujourd'hui le coming out, l'exposition de son orientation sexuelle, n'est pas de mise et hors de propos. Les cultures visuelles et orales d'avant les revendications identitaires et les luttes pour les droits, c'est-à-dire d'avant la visibilité homosexuelle, n'opèrent pas à découvert à la télévision ${ }^{19}$. Elles se construisent de significations codées et cachées, qui ne sont ni universelles ni intemporelles, pas plus que ne le sera l'identité gay et/ou lesbienne : nous nous sommes engagées à leur recherche. 


\section{Corpus et méthode}

4 Le corpus que nous avons délimité au sein des émissions de divertissement télévisuel ayant été diffusées entre 1955 et 1969 relève d'une source unique d'archives : ce sont celles que conserve l'Institut national de l'audiovisuel (Ina). Celles-ci, pour la période des débuts de la télévision française, sont constituées des documents qui ont été diffusés sur support film ou enregistrés par kinéscope, dispositif permettant de fixer sur pellicule cinématographique les images transmises sur l'écran en direct pour les rediffuser ou les conserver. Dès $1949^{20}$, constatant le besoin de réutiliser des documents pour les réinsérer dans des journaux télévisés ou de rediffuser une émission, on a commencé à conserver les «bobineaux films » du journal. Un début de cinémathèque naît en 1952: on y retrouve à partir de 1958 les actualités, les productions ${ }^{21}$ de la Radiodiffusion-télévision française (RTF, puis ORTF pour Office de radiodiffusion télévision française) et les kinéscopes. L'Ina a été chargé de la conservation de ces fonds d'archives au moment du démantèlement de l'ORTF en 1974. Ces documents sont désormais consultables à l'Inathèque. En plus des émissions de variétés, nous avons aussi choisi d'intégrer dans notre corpus quelques extraits de journaux télévisés ou du Magazine féminin où apparaissaient les personnages et les signes récurrents du récit qui a commencé à se tisser devant nos yeux. Nous avons également regardé la presse de télévision née au tout début des années $1950^{22}$, en particulier Radio-Cinéma et RadioTélévision, pour y retrouver les articles présentant ou accompagnant les émissions de notre corpus. Enfin, nous avons procédé à une collecte non systématique de pochettes de disques ( 45 tours notamment) et de partitions de chansons associées, elles aussi, aux personnages récurrents des émissions télévisées considérées.

5 À partir de ces fonds méconnus que nous avons rassemblés, nous avons procédé au visionnage de l'ensemble du corpus. Nos recherches ont porté sur les personnes représentées (leur nom, leur qualité - chanteuse ? auteure ? les deux ?) et nous avons analysé le nombre de caméras utilisées et leur disposition, le décor, la mise en scène et les références qu'elle peut convoquer. Les émissions de variétés de la période choisie tressent deux fils rouges dans leur continuité, celui du "numéro " (chanson, danse, etc.) et celui de la " conversation ». Il nous importait de les compléter par des aperçus de la « vie privée » des présentatrices comme des invité.e.s, permettant de trouver et de comprendre leurs liens et, le cas échéant, de tracer l'esquisse d'un réseau renvoyant à une histoire culturelle non patriarcale et non hétérosexuelle. Tel est le rôle qu'ont joué pour nous certaines images des magazines de programmes, souvent des mises en scène photographiques de la vie "hors télévision » de telle présentatrice, comédienne ou chanteuse. De même, les pochettes de disques et les partitions de chansons ont été utilisées pour confirmer ou infirmer les présentations de soi qu'induisait le visionnage des émissions. Nous avons considéré les images, les textes des chansons et enfin les crédits pour repérer les signes et les circulations récurrentes dans une culture homosociale "pré-Stonewall », c'est-à-dire d'un temps antérieur à celui où l'on s'identifie publiquement comme lesbienne, gay ou trans.

6 Notre recherche prolonge celle qu'a tentée le théoricien américain David M. Halperin dans son "Art d'être gai» (How to be gay, en langue originale), qu'il a ancré sur des pratiques et non sur des personnes.

Pour comprendre la culture gaie, on doit s'intéresser non à des types de gens (homosexuels ou non) mais à des types de discours et des types d'interactions, quels 
qu'en soient les sujets. Notre objet d'études, c'est la culture gaie, pas les gais, pas l'identité gaie. [...] L'objectif immédiat est de mettre en lumière quelques caractères pragmatiques de la culture gaie et de décrire les formes de l'expérience subjective ou les structures collectives de perception que des façons particulières d'agir et de communiquer expriment ou produisent chez ces gens, gais ou non, qui participent à

la culture résultant de ces pratiques génériques ${ }^{23}$.

Comme David M. Halperin a tenté de le faire pour la culture homosexuelle masculine anglophone et particulièrement américaine, nous nous proposons non pas d'étudier la culture lesbienne francophone dans son ensemble, mais de tenter de produire, à partir de matériaux télévisuels, photographiques, journalistiques et musicaux, un répertoire sémantique en usage dans les années 1950-1960. Pour cela, nous faisons appel au registre des études visuelles, qui nous permettent d'analyser et d'interpréter un corpus de documents audiovisuels, de façon pragmatique, document par document. Les questions du regard, de l'adresse et du public y sont centrales. Nous ajoutons à cette analyse la grille de lecture des queer studies. « Queer souligne [...] ce qui ne se laisse pas assimiler par la vie normale, ce qui brise les normes acceptées ou s'en tient à l'écart ${ }^{24}$. » L'utilisation d'une approche queer consiste ainsi à repérer et à se laisser guider par « ce qui ne va pas ", ce qui « cloche », ce qui fait «bizarre ». Ce décalage qu'on peut déceler jusque dans la fluidité des rôles joués par les protagonistes des émissions de variétés (présentatrices, productrices, auteures, compositrices, interprètes...) nous invite à reconstituer un "positionnement queer» dans l'espace public, dans l'espace médiatique, et à y reconnaître une culture lesbienne, en un temps où les personnes qui $y$ interviennent ne se seraient jamais reconnues dans ce nom ${ }^{25}$.

\section{Le cabaret télévisuel de Colette Mars et de Micheline Sandrel}

8 Dès 1946, la cartographie des cabarets de Paris s'enrichit en densité. Il est donc logique que la télévision française offre le cabaret au public des téléspectateurs.trices, qui se constitue en même temps que ce nouveau média en demande de programmes ${ }^{26}$. Un transfert quasi littéral s'opère de la ville à la scène des studios. Le Cabaret $d u$ soir ouvre ses portes durant deux ans le samedi, pendant une demi-heure, en dernière partie de soirée ${ }^{27}$. La journaliste Micheline Sandrel ${ }^{28} \mathrm{y}$ est associée à la chanteuse et comédienne Colette Mars : en duo, elles produisent et présentent cette émission. Colette Mars est une transfuge officieuse du monde de la nuit interlope, où se manifestent des genres et des sexualités équivoques ${ }^{29}$. Au Cabaret du soir, le genre féminin l'emporte de façon manifeste. Ce sont en effet de façon prédominante des chanteuses (Barbara y fera ses débuts télévisés), comédiennes ou personnalités féminines à la mode qu'elles y reçoivent, ainsi que de nombreux numéros de transformistes qui redondent, en les parodiant, les traits particularisés d'un féminin prééminent. Cette féminisation s'accomplit par le biais d'une écriture télévisuelle particulière, que l'on peut décliner comme suit.

9 En studio est reproduit le cadre supposé d'un cabaret « Rive droite » - pour reprendre la typologie traditionnelle d'une différence des rives de la Seine en deux classes, bourgeoise d'un côté, bohème de l'autre, quant à leurs types de spectacles comme à leur fréquentation. Le décor est composé de guéridons, dressés de nappes et munis de bouteilles de champagne et de coupes. Entre les chansons, les sketchs ou les numéros, les présentatrices passent d'une table à l'autre. L'utilisation de trois caméras permet de 
filmer frontalement les performances et de chorégraphier les transitions vers la table où s'engage la conversation avec quelques invité.e.s, mises en lumière. En arrière-plan, des tables de figurant.e.s - où on reconnaît nombre de jeunes comédien.ne.s, tel.le.s Judith Magre ou Jean-Claude Brialy ${ }^{30}$ - renforcent l'impression d'une continuité de lieu entre certains cabarets - où ils et elles pourraient parfaitement se trouver - et le plateau de télévision, qui s'efface. Après une répétition l'après-midi, le spectacle est produit en direct pour la France métropolitaine ${ }^{31}$, reproduisant les conditions et le déroulement en usage dans les cabarets. Une petite formation de trois à quatre musiciens, à peine aperçue par les téléspectateur.trice.s, accompagne les interprètes. Celles-ci peuvent aussi bien chanter assises à leur place que debout, quelques pas plus loin : la place pour les spectacles est réduite.

10 Le générique démarre, Colette Mars s'avance vers les téléspectateurs : cheveux courts, robe simple, ajustée aux gestes de la chanteuse-pas de New Look ni de décolleté vertigineux. Elle ne porte pas de bijoux (ou très peu), seulement une bague à l'auriculaire droit, que la télévision capte de façon insistante. Colette Mars interprète une de ses chansons récentes, «Si tu crois $\|^{32}$. Tout de suite après, Micheline Sandrel, à une table, commence une conversation, à laquelle viendra ou pas s'ajouter Colette Mars, intervenant parfois depuis une autre table, vers laquelle la caméra se dirigera. La complicité des deux femmes est palpable. Des tutoiements malencontreux viennent de temps en temps s'immiscer dans leurs conversations, qui remplacent ici le mode de l'interview ${ }^{33}$. L'esprit de la conversation est en effet à l'honneur, reprenant en mode mineur la sociabilité des salons de la III ${ }^{e}$ République où les femmes jouaient un rôle éminent pour le lancement d'un auteur, d'un peintre ou d'un musicien ${ }^{34}$.

\section{Les spectat.eurs.rices sémiologues : un contre-public télévisuel}

11 Mêlant le décor « Rive droite » et l'encombrement « Rive gauche », l'émission combine également les styles de performances caractéristiques de chaque rive. Ainsi chanteur.se.s et attractions proviennent plutôt de Saint-Germain-des-Prés ${ }^{35}$, tout en se mélangeant aux usages relationnels des cabarets à sensibilités lesbiennes de la «Rive droite " que représentent La Vie parisienne de Colette Mars, Chez Suzy Solidor ${ }^{36}$ et le Carroll's de Frédé où chante Dany Dauberson ${ }^{37}$, une invitée fréquente des variétés télévisuelles. De même, Cabaret du soir s'intéresse au jazz et à ses chanteuses noires comme aux cantatrices blanches de l'opéra ${ }^{38}$. L'émission invite les comédien.ne.s d'un théâtre ou du cinéma dit de "qualité française ${ }^{39}$ » qui règne sur l'après-guerre. Mais elle intègre aussi des duettistes ou des performers repérés dans la cartographie homosexuelle des cabarets d'avant-guerre, comme le sont les duettistes Brancato et Charpini, ce dernier chantant les rôles féminins avec une bonne dose d'autodérision ${ }^{40}$. Une fois, Charpini revient seul ${ }^{41}$ pour évoquer deux anecdotes troublantes sur les réactions de son père et de la mère de Brancato à la vision de leurs spectacles: elles sont destinées à déclencher un rire de connivence auprès d'un public hétérosexuel. Cependant, elles résonnent douloureusement pour d'autres téléspectateurs.trices, qui y reconnaissent les signes d'un rire homophobe, c'est-à-dire d'un rire " de persécution " par lequel ils et elles se sentent désigné.e.s et identifi.é.s. Le spectacle s'adresse à tous et toutes, mais il y a celles et ceux qui savent lire "entre les lignes». Cette terminologie, empruntée au politologue Leo Strauss, et transposée de l'art d'écrire au spectacle du petit écran, permet de caractériser le partage qui s'opère au sein du public télévisuel : 
La persécution donne ainsi naissance à une technique particulière d'écrire et par conséquent à un type particulier de littérature, dans lequel la vérité sur toutes les questions cruciales est présentée exclusivement entre les lignes. Cette littérature s'adresse, non pas à tous les lecteurs, mais seulement au lecteur intelligent et digne de foi. Elle a tous les avantages de la communication privée sans avoir son grand désavantage-n'atteindre que les relations de l'écrivain. Elle a tous les avantages de la communication publique sans avoir son plus grand désavantage - la peine capitale pour son auteur ${ }^{42}$.

Cabaret du soir, émission calquée sur un univers (un contexte, des personnalités qui l'animent et le peuplent) dont les connotations font déjà référence à un corpus d'expressions homosociales, permet aussi une lecture « entre les lignes ». Cette lecture est adressée à un public averti et sémiologue, puisqu'il sait percevoir des affects divergents et dissidents dans le flux télévisuel produit et diffusé en direct. Ce « contrepublic $^{43}$ " se forme ainsi avec et contre la culture de masse dont la télévision est le véhicule. C'est en sa compagnie qu'il faut relire et réviser à notre tour de nombreux moments télévisuels. On peut se laisser guider, par exemple, par les performances de genre récurrentes de l'imitateur Claude Véga, qui lorsqu'il parodie La Callas, le 3 janvier 1959, laisse libre cours à une interprétation très « folle » de son personnage. Un art de la lecture est ainsi rendu possible par une écriture télévisuelle qui, pour paraphraser Monique Wittig, « annule les genres en les rendant obsolètes ${ }^{44}$ ».

\section{Les cousines : variations sur une parentèle queer}

Du côté des femmes, la scène la plus troublante de ce cabaret a lieu le 15 février 1958, alors que Micheline Sandrel et Colette Mars entourent la célèbre antiquaire du 20, rue du Faubourg-Saint-Honoré, Yvonne de Brémond d'Ars. Bague au petit doigt, cheveux et veste de coupe masculine (quoique cette dernière fût agrémentée de pierreries), elle est flanquée de sa cousine, la comtesse Anatole de Brémond d'Ars, version féminisée de la première. La conversation nous apprend qu'Yvonne et sa cousine vont prochainement à Londres pour le tournage d'un film tiré d'un des livres de l'antiquaire ${ }^{45}$. Yvonne et sa cousine surenchérissent sur ce voyage à deux. A priori, rien de particulier si ce n'est l'approbation souriante des quatre femmes à cette information de deux cousines partant à Londres. Cependant une autre lecture est possible pour certaines téléspectatrices. Yvonne de Brémond d'Ars est connue dans le milieu homosexuel. On sait sa longue relation avec Suzy Solidor, qu'elle "sculpta» de 1918 à $1930^{46}$. La comtesse Anatole de Brémond d'Ars a remplacé celle-ci dans le cœur de l'antiquaire et, opportunément mariée à un parent proche d'Yvonne, travaille au magasin d'antiquités de concert avec son amie, comme on peut le voir dans trois autres sujets télévisés de la $\mathrm{RTF}^{47}$. C'est bien un couple de femmes qu'interviewent nos deux présentatrices. Si elles sont cousines par alliance, en s'appelant mutuellement « cousine », elles utilisent aussi un terme qui longtemps a permis aux femmes vivant en couple de donner un lien de famille aux yeux extérieurs à la personne qui partageait leur vie, sans le justifier le plus souvent ${ }^{48}$.

\section{L'inquiétante étrangeté des regards}

14 Une forme de relationnalité « entre femmes " s'installe dès la première émission, le 14 septembre 1957, lorsque Colette Mars interpelle la comédienne Andrée Debar à propos de chapeaux, celle-ci annonçant comme en aparté qu'elle déteste ceux que lui fait 
porter Jacqueline Audry. En fait, elle fait allusion au film La Garçonne $e^{49}$, dans lequel elles jouent deux personnages qui ont une liaison. La télévision, via l'émission de variétés 36 chandelles ${ }^{50}$, a déjà montré Andrée Debar présentant les acteurs du film et Colette Mars interprétant, en " garçonne », la chanson éponyme. Dans ces deux moments télévisuels, il n'est aucunement fait allusion à l'homosexualité des personnages, qui est néanmoins évidente pour qui connait le roman de Victor Margueritte.

Autre comédienne de Jacqueline Audry, Edwige Feuillère est invitée au Cabaret du soir ${ }^{51}$. L'actrice, populaire depuis L'Aigle à deux têtes de Jean Cocteau (1946), a eu le courage de jouer dans Olivia, en $1951^{52}$, un rôle délicat : à la tête d'un pensionnat de jeunes filles, elle forme un couple avec "Mlle Cara» (Simone Simon). Jacqueline Audry est la première depuis l'avant-guerre à oser aborder le sujet des amours féminines. Tout aussi teintée d' "inquiétante étrangeté ${ }^{53}$ est la conversation qui se négocie sur le plateau entre Edwige Feuillère, Colette Mars et Paule Le Métayer. Manifestement, toutes trois se connaissent bien : Edwige Feuillère explique qu'elle porte régulièrement des bijoux fabriqués par "Mlle Le Métayer» (le fait qu'elle soit demoiselle est ainsi mis en évidence), Colette Mars ajoute avec enthousiasme que Mlle Le Métayer est aussi auteure de poèmes et engage Edwige Feuillère à lire l'un d'entre eux. En actrice consommée, Feuillère fait une lecture magistrale d'un texte qui a pour atout d'être suffisamment ambigu pour que, à l'écoute, il soit impossible de savoir la nature des amours en question.

16 Lorsqu'elle lit le poème, les regards de l'actrice se font attentifs à la place de la caméra. Edwige Feuillère la regarde «en comédienne » consommée qui, parce qu'elle a joué au cinéma, sait ce que la caméra renvoie de son image. Ces regards caméra, cependant, sont tabous au cinéma : ils ne se produisent jamais qu'à la télévision, et uniquement durant le temps où les chanteuses exercent leur métier, qui consiste à chanter, comme au cabaret, pour un public face à elles. Dès qu'elles rejoignent le plateau des invitées, elles semblent emportées dans le présent de la conversation. Les regards partagés, alors plus complices, témoignent d'un autre temps. On doit ici rappeler que le direct est permanent et que, en l'absence de différé, les invitée.s ont peu d'éléments de ce que voient les téléspectatrices.eurs. Il leur est difficile de se rendre compte de ce que leur regard pourrait trahir, lorsqu'il se fait complice ou un peu trop appuyé sur une silhouette ou un visage. À la fin des années 1950, le média télévisuel ne propose pas encore de retour - un moniteur qui permettrait de se voir au présent, sur le plateau - et ne permet pas de se "revoir ", une fois rentré.e chez soi. Avec le temps, durant les années 1960, la conquête progressive des foyers par la télévision et la compréhension progressive de ce que la caméra rend visible, les regards des invitées vont devenir neutres. Il ne faut pas laisser prise à d'autres lectures moins complices. Bientôt, chacun.e va regarder la caméra comme un.e professionnel.le de la télévision.

\section{Un « entre femmes » en voie de disparition}

17 Ce que nous voyons grâce à ces deux productrices-présentatrices est donc un dispositif propre à cette période d'expérimentation télévisuelle de l'avant 1960. Il propose un « entre femmes » où les hommes ne sont pas automatiquement mis au centre du propos ou de l'adresse et où les femmes se regardent, s'écoutent, s'admirent, se soutiennent. $\mathrm{Au}$ Cabaret du soir, cette "homosociabilité » a encore une place, visible ou invisible suivant les regards. C'est aussi ce que nous disent les yeux moqueurs et admirateurs de 
l'écrivaine Christiane Rochefort ${ }^{54}$. Invitée pour son livre Le repos du guerrier ${ }^{55}$, qui a reçu le prix de la Nouvelle Vague, elle est assise entre Micheline Sandrel et Colette Mars. Ses regards vont de l'une à l'autre, mi-séduite, mi-ironique, alors que Colette Mars l'incite à venir plus souvent «au cabaret». Christiane Rochefort, prise par l'ambiance, se lance dans une comparaison scabreuse : «Je serai comme le roi Pausole, une femme à la fois mais toute l'année ${ }^{56}$.» Au sein des énoncés équivoques que donnent à entendre ces moments télévisuels, il faut aussi compter avec les chansons «intersexes ${ }^{57}$ ", qui font dissidence par rapport à la binarité marquée des pôles féminin et masculin. Par exemple, lorsque Colette Mars chante Garde ça pour toi ${ }^{58}$, le morceau ne se départit jamais de son ambiguité pour évoquer un amour qu'il faut garder secret, car «le mauvais cœur des hommes n'y comprendrait rien du tout ». La chanteuse veille à inclure tout genre d'auditeur.trice.s. Au cabaret, un regard appuyé, une rime accentuée peuvent suffire à changer l'adresse d'une chanson et c'est ce dont Cabaret du soir prend acte.

\section{Le monde des variétés}

L'après-guerre en France signifie pour les femmes le retour vers un foyer imaginaire. La pression est forte, même dans le milieu ouvrier, pour fonder la cellule familiale idéale : le père au travail, la mère au foyer et les deux enfants. Les gaullistes, les communistes s'y mettent pour consacrer un modèle pourtant déjà pris en défaut : de plus en plus de jeunes femmes font des études, travaillent. Citoyennes munies du droit de vote depuis 1944, les Françaises de métropole savent déjà qu'elles ne correspondent pas à l'idéal projeté. Certaines vont essayer de s'y conformer, d'autres inventent des stratagèmes pour y échapper sans choquer. Ce que montre aux téléspectatrices la télévision des années 1950 et 1960, c'est l'éventail des possibilités d'être une femme : à condition de garder les apparences, tout semble possible. La télévision est alors façonnée par nombre d'anciens résistants et de jeunes gens attirés par les possibilités de cette nouvelle technologie. Pour ces raisons, des femmes y trouvent leur place, la télévision n'étant pas encore un lieu de pouvoir. Au public, la télévision veut offrir une fenêtre pour aller ailleurs, chez les autres, pour découvrir tout ce à quoi il n'a pas accès : la France, les colonies ${ }^{59}$, le monde, différents milieux et cultures. Plus que tous les autres genres, les variétés édifient en modèles des chanteur.se.s, et des comédien.ne.s. Brigitte Bardot est certes apparue comme le canon du féminin par excellence de cette époque, populaire et d'avant-garde. Pourtant, à regarder dans les marges de la culture - les journaux de programme télévision, les revues de cinéma populaire, les partitions de chansons, les pochettes de disques 45 tours -, on découvre aussi d'autres constructions genrées, annonçant d'autres subjectivités ${ }^{60}$, d'autres façons d'être et de se vivre face à la cellule familiale.

\section{Quand les attitudes deviennent formes}

19 Ainsi déboule dès 1952 sur le petit écran la chanteuse Mick Micheyl, avec sa tignasse courte et bouclée. Dans les émissions de variétés - Cabaret du soir, École des vedettes ou 36 chandelles -, Mick Micheyl est vêtue, de façon récurrente, d'une robe sans manche coupée dans un tissu sombre, d'où émerge un énorme col de chemise blanc façon «pelle à tarte». Celui-ci, les pans relevés, entoure son visage comme le ferait une 
collerette sur un pourpoint masculin. C'est sa marque de fabrique, figurant sur les photos d'une large partie de ses pochettes de disques 45 tours, entre 1954 et 1961. Quand on la parodie, comme le fait l'imitateur Claude Véga, on fait le geste de remonter son col $^{61}$. Son visage simplement entouré de ces deux ailes blanches suffit pour la définir. Elle ne porte généralement pas de bijou, parfois simplement une bague au doigt ou une médaille autour du cou, parfois une montre ressemblant à un bracelet de force. Mick Micheyl adopte ainsi, dans la première partie de sa carrière, une forme de compromis vestimentaire. Celui-ci rejoue parodiquement les enjeux de l'habit noir ${ }^{62}$ endossé par l'homme bourgeois à partir du XIX $x^{e}$ siècle, mais sous une forme revue et corrigée par les déclinaisons d'un dandysme féminin, qu'on retrouve dans certaines représentations de l'entre-deux-guerres. Ainsi de La Duchesse de La Salle (1925) ${ }^{63}$ peinte par Tamara de Lempicka, sur un escalier et en costume de cheval, chemise ouverte au col relevé, une main nonchalamment rentrée dans la poche de son pantalon. Ou encore de l'Autoportrait (1923) peint par Romaine Brooks ${ }^{64}$ : chapeau claque enfoncé jusqu'aux yeux, elle porte l'habit noir et le col de chemise ouvert et relevé, deux ailerons blancs se détachant de la masse sombre de l'habit et du couvre-chef. Son visage, dont les yeux sont à demi cachés, est poudré et ses lèvres, maquillées de rouge. La représentation, combinant des traits associés au féminin et d'autres au masculin, se détache ainsi de toute authenticité par rapport à une " vraie nature ${ }^{65}$ ", pour devenir celle d'une femme moderne hors de l'économie patriarcale, du repère hétérosexuel et de l'assimilation du masculin à un homme. Plus tard, Mick Micheyl adoptera aussi la tenue tout en plumes de la meneuse de revue du Casino de Paris. Voir la chanteuse adopter alternativement à la télévision le style d'une "masculinité sans homme » et celui d'une "féminité sans femme ", c'est aussi inscrire les modalités d'énonciation des marques du genre dans une modification et une fluidité permanentes.

\section{Mémoires d'une jeune fille dérangée}

L'image nouvelle de la jeunesse qui "va mourir demain ${ }^{66}$ » s'incarne à la télévision dans le succès de Gribouille. Dès son premier passage à l'écran ${ }^{67}$, elle se présente vêtue d'un pull de laine beaucoup trop grand pour elle, dans une figuration androgyne à la moue butée et au regard défiant de la vagabonde sans famille. Elle dessine sur le sol. Elle se présente : "Gribouille. » La caméra fixe l'une de ses "craies", reprenant les dessins de têtes d'enfant qu'elle trace ${ }^{68}$ sur les trottoirs de Saint-Germain-des-Prés. On dit que Jean Cocteau, flânant dans Paris, s'est mépris sur son genre, l'a prise pour un garçon et l'a recommandée au cabaret Le Bœuf sur le toit, où elle a trouvé l'un de ses premiers engagements ${ }^{69}$. Ce sont ses débuts dans le monde télévisé : «Et que voulezvous maintenant?», lui demande le comédien Jean-Pierre Aumont. "Je voudrais chanter ", répond-elle. Gribouille interprète Le Marin et la rose, un morceau directement hérité de Suzy Solidor, qui le chantait à La Vie parisienne en 1933. La télévision est sous le charme: pendant les quelques années avant sa mort en 1968, à l'âge de 27 ans, Gribouille n'apparaitt pas moins de quatorze fois et chante son célèbre Mathias cinq fois. Les barreaux aux fenêtres qui constituent le décor de sa première apparition se répètent, sous forme d'ombres menaçantes, dans les cadres des images ultérieures. Ces frontières spectrales composent comme une grille autour de sa silhouette sombre debout, statique, alors que ses mains s'érigent en témoin d'une tristesse râpeuse, celle d'un Cour en berne, qui attend. Elle t'attend : le féminin est choisi sans équivoque. Assise, dos à la caméra, sur une simple chaise pliante, dans un studio nu peuplé de fantômes, 
elle se lève, en pantalon, chemise et pull-over avec ses mèches courtes sur son front. Pour la première fois peut-être, de façon à peine voilée à la télévision, Gribouille propose dans sa performance une adresse assez directe pour réveiller les fantasmes et les désirs de spectatrices qui la reconnaissent et se reconnaissent. En d'autres termes, Gribouille parle, au sein de l'espace public du média télévisuel, « aux lesbiennes ».

\section{Le col Claudine}

21 "Quand et si je me dévoile comme lesbienne, qu'est-ce qui est "out", qui se rend manifeste et se révèle complètement? Qu'est-ce qui se sait alors, est-ce la même quelque chose $^{70}$ ?» Nicole Louvier s'affiche dans la lignée des Claudine ${ }^{71}$, la série imaginée par Colette : une jeune personne timide et effrontée, vivant avec sensualité l'éveil des sens, liseuse vorace et sans famille. Elle a les cheveux courts, le regard lourd de sous-entendus, le sourire charnu, le col de son chemisier blanc boutonné sous son pull, la guitare en main. À 20 ans à peine, elle a publié, tout en composant des chansons, le roman Qui qu'en grogne (1953) ${ }^{72}$. Il est le récit à la première personne du rêve d'une jeune fille pour une autre, de leur amour et de sa fin; un amour au terme duquel la possibilité d'être attirée par d'autres jeunes femmes promet une alternative au couple hétérosexuel. La même année, Qui me délivrera (1953), qu'elle donne d'abord à la chanteuse Noëlle Norman, obtient le grand prix au concours de la chanson française à Deauville. "Qui me délivrera / De ton corps de tes doigts? / De ta bouch'qui me délivrera? Qui me délivrera / Enfin de toi?» Sur un tempo di bolero ${ }^{73}$, les paroles laissent planer l'équivoque quant au genre $\mathrm{du}$ « tu » qui est l'objet de la plainte et du désir lancinants. Et lorsque Nicole Louvier apparaît à la télévision ${ }^{74}$, c'est précédée de l'aura de Qui qu'en grogne et de la publication d'un volume de poèmes érotiques qui " chantent l'amour homophile, celui de Lesbos ${ }^{75}$ », sous le titre de Chansons interdites. On la retrouve, comme de bien entendu, dans l'émission 36 chansons $^{76}$ en 1957 et dans le partage intimiste d'une table à quatre femmes composant le menu d'un Cabaret du soir ${ }^{77}$. Colette Mars la complimente avec un ton qui dépasse l'admiration: "J'adore votre voix », lui dit-elle.

\section{Le lit à une place}

Une autre façon de signifier cet aparté des jeunes femmes sans homme est d'insister sur un mode d'existence qui se traduit par la mise en scène d'un lieu de vie : l'appartement (le studio) célibataire. Chambre à soi, il certifie le vécu autonome d'une femme, comme l'avait prôné Virginia Woolf. Mais lorsqu'il apparaît dans la lumière télévisuelle, il permet de composer avec des indices d'un écart par rapport à la norme sociale de l'époque : celle qui lie le domestique, le familial, le féminin matrimonial et l'obligation à l'hétérosexualité. Dans un Magazine féminin ${ }^{78}$, Nicole Louvier est interviewée "chez elle». Le cadre est encombré d'un piano droit, d'un mur orné d'une affiche en caractères japonais, d'une guitare et d'un petit lit où elle est assise avec son interlocutrice, Micheline Sandrel. D'émission en émission, la chanteuse-écrivaine fait obligeamment la visite de sa cuisine, de sa chambre, de sa salle de bains, toutes à l'échelle «je vis seule». Le lit à une place certifie l'absence de conjugalité aussi bien hétéro- qu'homosexuelle: on le devine, le studio célibataire est aussi la figure d'un compromis avec la morale. Ainsi Nicole Louvier peut-elle ouvrir son placard: "Les vêtements de femme, c'est terrible, ça prend de la place. [...] Je n'aime pas qu'on 
m'oblige à me déguiser ${ }^{79} ! "$, s'exclame-t-elle. La dénaturalisation qu'elle opère dans son discours renvoie à une autre "nature " dans laquelle elle baigne : le paysage composé de ses disques, de ses livres, de sa télévision, de son chien. Ce paysage organise un discours adolescent et consumériste. La médiatisation, par la télévision, d'un appartement ainsi muni des signes caractéristiques d'une vie de (femme) célibataire autonome dans ses choix est exactement contemporaine de celle du programme "d'utopie sexuelle, post-domestique et urbaine ${ }^{80}$ » imaginé par Hugh Hefner dans le magazine américain Playboy, pour transformer l'homme hétérosexuel américain en playboy. L'opérateur de cette transformation, c'est ainsi que l'a théorisé Paul B. Preciado, est l'invention d'un topos érotique alternatif au pavillon de banlieue familial, inoculé d'abord "par le biais du magazine, puis par celui de la télévision, un espace virtuel [déployé] par le texte et l'image ${ }^{81} »$. Certes, l'architecture playboy est loin des représentations sages de l'appartement dans lequel la télévision persiste à placer Nicole Louvier. C'est plutôt par élision et dénégation qu'un tel décor désigne en creux la célibataire sans homme et, au-delà de cette dénomination, laisse imaginer un corps qui s'en passe pour trouver son plaisir. Lorsque Nicole Louvier chante Nicole Louvier ${ }^{82}$, le cadre aussi reconstitué sur un plateau de télévision est celui d'un appartement qui semble s'être transporté avec elle. Cette reterritorialisation, qui s'inscrit aussi dans le mouvement d'urbanisation des années 1960, caractérise également une subjectivité qui trouve refuge hors de la sphère familiale et domestique de la féminité.

\section{Masculinités féminines}

En l'absence d'études historiques ou anthropologiques en langue française sur les lesbiennes dans la période que nous considérons, nous nous reportons aux travaux existants aux États-Unis ${ }^{83}$ sur l'histoire des homosexuel.le.s entre la Seconde Guerre mondiale et la révolte de Stonewall en 1969. Nombre d'entre eux ont mis l'accent sur un phénomène urbain particulier aux lesbiennes de la classe ouvrière dans les grandes villes industrielles des États-Unis. Il s'agit du couple butch-fem(me) : deux termes codés, qualifiant d'une part les femmes qui se sentent plus à l'aise ${ }^{84}$ en utilisant les codes du masculin, le pantalon, les métiers d'hommes; et de l'autre, les femmes qui parodient de la même façon les codes du féminin. Pourtant, ce phénomène, qui affecte les communautés blanches et noires, reste attaché à une notion de classe sociale. Au sein d'une sphère plus privilégiée économiquement, les femmes qui aiment les femmes font certes appel à des signes et des styles vestimentaires reconnaissables, mais sans en référer à ce "marquage " butch/fem. C'est aussi ce qu'on peut constater dans les émissions de variétés de la télévision française, dont les protagonistes semblent tou.te.s appartenir à une même catégorie sociale (ou à ses variantes " artiste » ou «bohème ») et à un même genre, plutôt féminin. Cependant, la façon de bouger, de se mouvoir ou de porter la robe de cocktail rend visible un « malaise » dans la présentation corporelle de certaines de ces femmes. Dans l'émission de variétés Les Joies de la vie ${ }^{85}$ consacrée à la comédienne et chanteuse Odette Laure apparait celle qui lui donna son premier emploi, au cabaret La Vie parisienne : la blonde Suzy Solidor, la cinquantaine sonnée, cheveu milong, debout près d'un piano, dans une robe décolletée, dégageant les épaules, un simple bijou au cou, une étole tombant sur les bras, dressée comme une figure de proue. Elle semble parée des atours de la féminité : le maquillage (mais simple), la robe (mais simple), l'étole (mais qu'elle broie avec vigueur), autant d'éléments nécessaires à celle qui joue le jeu de son milieu et de la télévision, pour apparaître en conformité 
avec les assignations de genre en vigueur. Pourtant, quelque chose cloche dès que la main se tend et qu'apparaît une énorme chevalière à l'auriculaire, symbole d'une autre histoire pour qui sait voir. Quelque chose cloche quand, chantant l'histoire de la voleuse et du filou d'une voix grave, elle choisit la force bandée d'un bras, le haussement d'épaules musclées, la désinvolture du filou. Avec sa grande taille, Dany Dauberson semble quant à elle plutôt encombrée de la robe bustier échancrée dont elle se revêt lors d'un de ses passages au Cabaret du soir ${ }^{86}$. Elle accompagne de gestes amples et de mouvements affirmés sa chanson : «Si je pouvais revivre un jour... je saurais bien te dire que je t'aime / À toi pour qui je ne l'ai jamais dit. » La déclaration résonne pour celles qui, derrière leur téléviseur, gardent leurs amours secrètes sans oser les dire. Dany Dauberson est d'ailleurs l'une des seules à risquer la masculinité à la télévision ${ }^{87}$. Durant le temps d'une chanson, elle se revendique de son engagement de guerre en tant que soldate dans l'armée américaine et apparaît ainsi en G.I., assise solitaire à califourchon sur une chaise près d'un bar, pour entonner de sa voix grave Stormy Weather ${ }^{88}$, le tube météorologique du Cotton Club qu'ont chanté Lena Horne et Billie Holliday.

\section{La télévision et le secret du placard ${ }^{89}$}

La télévision introduit dans le chez-moi l'ailleurs, et le mondial, à chaque instant. Je suis plus isolé, plus privatisé que jamais, avec chez moi l'intrusion en permanence, par moi désirée, de l'autre, de l'étranger, du lointain, de l'autre langue. Je la désire et en même temps, je m'enferme avec cet étranger, je veux m'isoler avec lui, sans lui, je veux être chez moi ${ }^{90}$.

Caractérisée par Jacques Derrida comme média « échographique », la technologie de la télévision a introduit un nouveau rapport au monde : celui de l'apparition du monde " en direct ", le lointain surgissant en proche, l'absent.e figuré.e en présent.e, le mort en vif. Elle transforme en flux toute expérience intime, redistribuée au public par le média télévisuel reçu chez soi. La télévision construit ainsi « un savoir identifié en même temps comme secret (reçu à la maison) et partagé (défini comme participant à la culture nationale collective $)^{91}$. "

Dans le champ télévisuel des années 1955 à 1969, les variétés ont très vite été fédératrices: acceptant une grande liberté de ton, tout en repoussant loin d'elles les clivages politiques, religieux, raciaux et sexuels. C'est là que nous avons choisi notre corpus. Colette Mars et Micheline Sandrel sont apparues comme des évidences : l'une pour ses liens avec le music-hall et les cabarets interlopes, l'autre pour ses nombreuses interviews de femmes dans le Magazine féminin de la télévision. Au Cabaret du soir, leur émission, des signes historiques, culturels et factuels se sont multipliés, qui nous ont interpellées. Il nous suffisait de les relier les uns aux autres, de suivre certaines de leurs invitées pour découvrir les marges d'une féminité convenue et, avec elles, les prémisses d'une autre sociabilité, d'autres performances de genre, voire d'un trouble du genre. D'un "entre femmes", nous sommes passées à la mise en place d'un paradigme adolescent et ambigu qui accepte le féminin et le masculin comme une mascarade dont il faut se jouer pour rester libre, alors que d'autres choisissent de s'adresser plus directement à celles qui se reconnaitront comme elles, lesbiennes. Ce mot, comme celui d'homosexuelle, n'a alors pas de sens pour elles. Les années 1950 et 1960 convoquent plus subtilement des traces, qui sont autant de clefs ouvrant des modes de vie réservés jusque-là à certains lieux, certains milieux. La bague portée au petit doigt, la chaînette 
à la cheville, le cou dépourvu de bijoux, la chemise au col relevé, le port de talons plats, la robe enfilée sur des épaules trop larges et la façon qu'a le corps de la porter, le pull à col roulé, le regard de connivence, la force des bras bandés qui se lèvent en un geste ample, les jambes campées en position debout, la voix grave qui s'oublie, les paroles équivoques... - chacun de ces indices détectés ne révèle certes pas la vérité d'une " identité lesbienne ", qui serait ainsi essentialisée. C'est plutôt ensemble qu'artefacts, postures, façons de s'adresser aux autres et doubles sens concourent à un "style ". Nous empruntons à nouveau ce terme à L'Art d'être gai de David M. Halperin, qui l'emploie quand il s'agit de barrer la route à « une vision simpliste de ce qui fonde les choix d'objets culturels gais ${ }^{92} »$. Le secret de leur séduction, continue Halperin, est à chercher non dans leur signification cachée, mais dans leur forme, leur style. C'est aussi ce que nous avons ressenti en scrutant, à notre tour, les émissions de variétés des années 1955 à 1969, relevant d'une époque qui n'est pas susceptible d'affirmation gaie, lesbienne, bi ou trans explicite. Pourquoi continuent-elles à susciter des réactions enthousiastes d'identification et de jouissance ${ }^{93}$, en des temps où la vie gaie, lesbienne, bi ou trans est sortie du placard? Il nous semble que les écarts et les décalages que nous avons perçus par rapport au "roman familial » de la télévision de masse, sans jamais être définis par un nom ou une identité stable, peuvent aussi servir collectivement à en faire un usage décalé. S'offre ainsi « le moyen d'échapper à l'anormalité singulière, liée à l'expérience personnelle, pour accéder à une anormalité universelle valable pour tous.tes [qui] promet un monde et pas une identitée ${ }^{94}$.

\section{BIBLIOGRAPHIE}

ALAIN, Jeannine, 1958. «Chansons interdites, de Nicole Louvier » Arcadie 54, revue littéraire et scientifique [en ligne], consulté le 14 octobre 2016. URL : http://culture-et-debats.over-blog.com/ article-chansons-interdites-de-nicole-louvier-par-jeannine-allain-116611513.html

BRÉMOND D'ARS, Yvonne (de). 1957. C'est arrivé en plein Paris, passionnante aventure d'antiquaire. Paris : H. Lefebvre.

BUTLER, Judith. 1993. «Imitation and Gender insubordination », in The Lesbian and Gay Studies Reader. ABELOVE, Henri, AINA BARALE, Michèle \& HALPERIN, David M. (dir.). New York/London :

Routledge, 307-320.

CARBONEL, Marie-Hélène. 2007. Suzy Solidor, une vie d'amour. Paris : Autres Temps.

CHAMBERS, Scott. 2009. The Queer Politics of Television. London : I. B. Tauris.

CHETCUTI, Natacha. 2010. Se dire lesbienne. Paris : Payot.

DE LAURETIS, Teresa. 2007. Théorie queer et cultures populaires. De Foucault à Cronenberg (trad. Marie-Hélène Bourcier). Paris : La Dispute.

DERINÖZ, Sabri. 2013. « La représentation de l'homosexualité dans les médias de la Fédération Wallonie-Bruxelles » [en ligne], consulté le 13 octobre 2016. URL : http://csa.be/system/ documents_files/2045/original/SD_20130513_rapport final_publ.pdf?136870682 
DERRIDA, Jacques \& STIEGLER, Bernard. 1996. Échographies de la télévision. Paris : Galilée et Ina. ÉRIBON, Didier. 2003. «F.H.A.R. », in Dictionnaire des Cultures Gay et Lesbiennes, ÉRIBON, Didier \& LERCH, Arnaud (dir.). Paris : Larousse, 196.

FADERMAN, Lilian. 1991. Odd Girls and Twilight Lovers: a History of Lesbian Life in XX ${ }^{\text {th }}$ C. America. New York : Columbia University Press.

FAUSTO-STERLING, Anne. 2000. Sexing the Body. New York : Basic Books.

GAILLARD, Isabelle. 2012. La Télévision, histoire d'un objet de consommation.1945-1985. Paris : CTHS/ Ina.

GONNARD, Catherine. 2012. " Mixité, non mixité : heurts et dialogues d'un mouvement autonome entre 1980-2000» Coordination Lesbienne [en ligne], consulté le 15 septembre 2016. URL : http:// www.coordinationlesbienne.org/spip.php?article186

HARVEY, John \& LEE, John. 1998 [1996]. Les Hommes en noir, du costume masculin à travers les siècles. Paris : Abbeville Press France.

HALPERIN, David M. 2015 [2012]. L'Art d'être gai. (trad. Marie Ymonet). Paris : Epel.

HOOG, Emmanuel. 2006. L'INA. Paris : PUF.

JOYRICH, Lynne. 2001. «Epistemology of the Console » Critical Inquiry [en ligne], 27(3), consulté le $1^{\text {er }}$ septembre 2016. URL: http://www.jstor.org/stable/1344216

KOHNEN, Melanie E.S. 2016. Queer Representation, Visibility and Race in American Film and Television. Screening the Closet. London/New York : Routledge.

KOSOFSKY SEDGWICK, Eve. 2008 [1990]. Épistémologie du placard. (trad. Maxime Cervulle). Paris : Amsterdam.

LAPOVSKY KENNEDY, Elisabeth \& DAVIS, Margaret D. 2014 [1993]. Boots of Leather, Slippers of Gold: the History of a Lesbian Community. New York : Routledge.

LATIMER, Tirza T. 2005. Women Together/Woman Apart. New Brunswick, New Jersey : Rutgers University Press.

LEDOS, Jean-Jacques. 2007. L'Âge d'or de la télévision.1945-1975. Histoire d'une ambition française. Paris : L'Harmattan.

LESSELIER, Claudie. 2000. « Formes de résistances et d'expression lesbiennes dans les années cinquante et soixante en France ", in Homosexualités, expression/répression, TIN, Louis-Georges \& PASTRE, Geneviève (dir.). Paris : Stock, 105-116.

LOUVIER, Nicole. 1953. Qui qu'en grogne. Paris : Gallimard.

MARTIN-FUGIER, Anne. 2003. Les Salons de la III République, Art, littérature, politique. Paris : Librairie académique Perrin.

MONEY, John. 1952. Hermaphroditism: An inquiry into the nature of a human paradox. PhD diss., Harvard University.

ORAIN, Marie-Thérèse. 2001. Gribouille. Je vais mourir demain. Paris : Christian Pirot.

PENET, Martin. 2006. « L'expression homosexuelle dans les chansons françaises de l'entre-deuxguerres : entre dérision et ambiguïté » Revue d'histoire moderne et contemporaine [en ligne], 53(4), consulté le 13 octobre 2016. URL : www.cairn.info/revue-d-histoire-moderne-etcontemporaine-2006-4-page-106.htm 
PRECIADO, Paul B. (Beatriz). 2011 [2010]. Pornotopie. Playboy et l'invention de la sexualité multimédia. Paris : Flammarion.

ROCHEFORT, Christiane. 1958. Le Repos du guerrier. Paris : Grasset.

ROLLET, Brigitte. 2007. Télévision et homosexualité. 10 ans de fictions françaises 1995-2005. Paris : L'Harmattan.

RUBIN, Gayle. 1992. « Of Catamytes and Kings: Reflections on Butch, Gender and Boundaries », in The Persistent Desire : a Femme/Butch Reader. NESTLE, Joan (dir.). Boston : Alyson Publications.

RUPP, Leila J. 1999. A Desired Past, a Short History of Same-Sex Love in America. Chicago : University of Chicago Press.

SANCHEZ, Maria C. 2001. Passing: Identity and Interpretation in Sexuality, Race and Religion. New York : New York University Press.

SANDREL, Micheline. 1962. Dix millions de témoins. Paris : Hachette.

SAUVAGE, Monique \& VEYRAT-MASSON, Isabelle. 2012. Histoire de la télévision française de 1935 à nos jours. Paris : Nouveau Monde.

SCOTT, Joan W. 1988. « Genre : une catégorie utile d'analyse historique » Les Cahiers du GRIF, Le Genre de l'Histoire 37-38: 125-153.

SCOTT, Joan W. 2012 [1999]. « Quelques autres réflexions sur le genre et la politique », De l'utilité du genre (trad. Claude Servan-Schreiber). Paris : Fayard, ch. 3.

STOLLER, Robert. 1978 [1955]. Recherche sur l'identité sexuelle. Paris : Gallimard.

STRAUSS, Leo. 2009 [1952, 1989]. La Persécution et l'art d'écrire. Paris : Gallimard.

WARNER, Michael. 2002. Publics and Counterpublics. New York : Zone Books.

WALKER, Lisa. 2001. Looking like What You Are: Sexual Style, Race and Lesbian Identity. New York: New York University Press.

WITTIG, Monique. 2007 [1982, 2001]. « Le point de vue universel ou particulier (avant-note à La Passion de Djuna Barnes) » La Pensée straight. Paris : Éditions Amsterdam, 112.

\section{NOTES}

1. Il en est ainsi de l'émission La Joie de vivre de Henri Spade et Robert Chazal. Sauvage, Monique \& Veyrat-Masson, Isabelle. 2012. Histoire de la télévision française de 1935 à nos jours. Paris : Nouveau Monde, p. 47.

2. Ledos, Jean-Jacques. 2007. L'Âge d'or de la télévision. 1945-1975. Histoire d'une ambition française. Paris : L'Harmattan, édition Kindle.

3. Michèle Arnaud, la productrice de l'émission qui partage les risques des facéties d'Averty, n'est jamais mentionnée.

4. Au-delà du coming out télévisuel de Jean-Louis Bory en 1975, d'Elula Perrin en 1977 et des premiers personnages gays dans des séries télévisées diffusées en France (Dynastie, version française à partir de 1983), il semble que «c'est à partir de 1995 que certaines représentations [des homosexuel.le.s] commencent à s'inscrire de façon plus fréquente dans le paysage audiovisuel français ». Rollet, Brigitte. 2007. Télévision et homosexualité. 10 ans de fictions françaises 1995-2005. Paris: L'Harmattan, p. 16. Voir aussi Chambers, Scott. 2009. The Queer Politics of Television. London : I. B. Tauris. 
5. Derinöz, Sabri. 2013. «La Représentation de l'homosexualité dans les médias de la Fédération Wallonie-Bruxelles »[en ligne], consulté le 13 octobre 2016. URL: http://csa.be/system/ documents_files/2045/original/SD_20130513_rapport\%20final_publ.pdf?136870682

6. À partir de 1955, les supports matériels conservés des émissions sont plus nombreux. 1969 marque le début de « l'après mai » où d'autres histoires commencent à s'écrire.

7. Cabaret du soir: l'émission est diffusée du 14 septembre 1957 au 24 janvier 1959, d'abord régulièrement tous les quinze jours, puis plus irrégulièrement : une seule fois en mai 1958, deux fois en juin 1958, une fois en juillet, septembre, octobre et novembre 1958, deux fois en décembre 1958 et janvier 1959, jusqu'à la dernière émission du 24 janvier 1959. État actuel de la collection à l'Ina : il manque 14 minutes à l'émission du 14 septembre 1957 et l'émission du 7 décembre 1957 est manquante. C'est donc 24 sur 25 émissions que nous avons pu visionner. Jean Kerchbron est le réalisateur de la plupart de ces émissions.

8. Penet, Martin. 2006. «L'expression homosexuelle dans les chansons françaises de l'entre-deuxguerres : entre dérision et ambiguïté » Revue d'histoire moderne et contemporaine, [en ligne], 53(4) : 122-123, consulté le 13 octobre 2016. URL : www.cairn.info/revue-d-histoire-moderne-etcontemporaine-2006-4-page-106.htm

9. Le Magazine féminin était le premier magazine féminin à la télévision, d'abord produit par Aimé Chabrerie et animé par Maïté Célérier de Sannois, programmé à partir de décembre 1946 sous le nom La femme chez elle, puis, à partir de 1951, de Pour vous madame, devenant enfin Magazine féminin en 1952, diffusé en hebdomadaire jusqu'à son arrêt en 1970. Documents conservés à partir de mai 1952.

10. L'usage de ce verbe n'est évidemment pas anodin, si l'on se réfère à la culture américaine du "passing » : la capacité d'une personne à être considérée en un seul coup d'œil comme membre d'un groupe social autre que le sien propre, tel que l'ethnie, la caste, la classe sociale, le genre, l'âge et/ou le handicap. Sanchez, Maria C. 2001. Passing: Identity and Interpretation in Sexuality, Race and Religion. New York : NYU Press.

11. La Joie de vivre d'Henri Spade et Robert Chazal, émission bimensuelle en alternance avec 36 chandelles de Jean Nohain, commence en 1952 (documents conservés à partir d'avril 1953) et s'arrête en septembre 1956, pour devenir Bouquet de joie jusqu'en décembre, puis Les Joies de la vie d'octobre 1957 à mai 1960. Les 36 chandelles ont été diffusées entre 1952 (documents conservés à partir de 1955) et juillet 1958.

12. École des vedettes, produite et présentée par Aimée Mortimer, est diffusée entre novembre 1956 et juillet 1963.

13. «Elle dut en outre procéder à ce dernier crève-cœur : céder son cabaret à Colette Mars, chanteuse dont elle avait inspiré le timbre de voix et avec qui elle avait eu une brève liaison. » Carbonel, Marie-Hélène. 2007. Suzy Solidor, une vie d'amour. Paris : Autres Temps, p. 240.

14. Discorama, émission hebdomadaire produite par Denise Glaser de février 1959 à janvier 1975. 15. Scott, Joan W. 1988. «Genre : une catégorie utile d'analyse historique » Les Cahiers du GRIF, Le Genre de l'Histoire 37-38 : 125-153 ; Scott, Joan W. 2012 [1999]. «Quelques autres réflexions sur le genre et la politique ", in De l'utilité du genre. Paris : Fayard, ch. 3.

16. Money, John, 1952, cité par Fausto-Sterling, Anne. 2000. Sexing the Body. New York: Basic Books, p. 46. Stoller, Robert. 1978 [1955]. Recherche sur l'identité sexuelle. Paris : Gallimard. On peut remarquer que Le Deuxième Sexe de Simone de Beauvoir est publié également en 1949.

17. Les premières réunions du Front Homosexuel d'Action Révolutionnaire (F.H.A.R.) et les premières manifestations contre l'homophobie datent de 1971. Éribon, Didier. 2003. «F.H.A.R.", in Dictionnaire des Cultures Gay et Lesbiennes, Éribon, Didier \& Lerch, Arnaud (dir.). Paris : Larousse, 196.

18. Cette différenciation s'opère vers 1981. «Les termes "lesbiens" et "gays" aux définitions plus politiques ont peu à peu fait disparaître les termes "homosexuels/lles". » Gonnard, Catherine. 2012. "Mixité, non mixité : heurts et dialogues d'un mouvement autonome entre 1980-2000» 
Coordination lesbienne [en ligne], consulté le 15 septembre 2016. URL: http:// www.coordinationlesbienne.org/spip.php?article186

19. Kohnen, Melanie E.S. 2016. Queer Representation, Visibility and Race in American Film and Television. Screening the Closet. London/New York : Routledge.

20. Hoog, Emmanuel. 2006. L'INA. Paris : PUF (coll. Que sais-je ?), p. 6-7.

21. Par le terme de production, on entend les créations de la télévision pour la télévision.

22. Les magazines de programmes de télévision ont peu à peu absorbé les journaux de programmes de radio nés après la Seconde Guerre mondiale. Entre 1947 et 1960, Radio-Cinéma devient Radio-Cinéma-Télévision, puis Radio-Télévision-Cinéma, enfin Télévision-Radio-Cinéma, puis Télérama ; entre 1957 et 1960, Radio-Télévision devient Télé7Jours. Télé Magazine est créé en 1955.

23. Halperin, David M. 2015 [2012]. L'Art d'être gai, trad. fr. Paris : Epel, édition Kindle, chap. 7, doc. 3. De telles études manquent encore dans les pays de langue française, notamment pour les années d'après la Seconde Guerre mondiale, où celle de Claudie Lesselier reste une exception. Lesselier, Claudie. 2000. «Formes de résistances et d'expression lesbiennes dans les années cinquante et soixante en France », in Homosexualités, expression/répression, Tin, Louis-Georges \& Pastre, Geneviève (dir.). Paris : Stock, 105-116.

24. Halperin, ibidem, préface à l'édition française, édition Kindle, doc. 1 .

25. L'enjeu sociologique de cette question est analysé par Chetcuti, Natacha. 2010. Se dire lesbienne. Paris : Payot.

26. «Le million de récepteurs est atteint en 1958, les dix millions en 1968. » Gaillard, Isabelle. 2012. La Télévision. Histoire d'un objet de consommation, 1945-1985. Paris : CTHS/Ina, p. 119.

27. Cf. note 7 .

28. Micheline Sandrel a suivi les cours de théâtre Charles Dullin durant quatre ans et a débuté à la radio en 1947, avant de devenir chroniqueuse au journal parlé.

29. Le cabaret La Vie parisienne, qu'elle dirige, est situé au 12 rue Sainte-Anne à Paris, une rue qui devint, après 1968, l'un des cœurs de la nuit gay. Voir « Colette Mars au cabaret », extrait du film Cargaison blanche de Georges Lacombe (1958) [en ligne], consulté le 13 octobre 2016. URL : http:// www.dailymotion.com/video/x17yilw_colette-mars_shortfilms

30. «Des familier.e.s du cabaret », comme le remarque un soir Colette Mars. Cabaret du soir, RTF, diffusion 12 octobre 1957.

31. L'enregistrement grâce au kinéscope permet un différé dans les pays francophones au Maghreb, au Canada, en Suisse et au Liban notamment, si l'on en croit Micheline Sandrel qui, dans son roman policier Dix millions de témoins, décrit très précisément une émission de télévision, produite et présentée par une chanteuse, qui a pour décor un cabaret. Sandrel, Micheline. 1962. Dix millions de témoins. Paris : Hachette, p. 29.

32. Cabaret du soir, RTF, diffusion 5 avril 1958.

33. C'est le reproche que feront certains critiques, accusant les présentatrices féminines de "papotage »: "Sur la bonne voie... Le Cabaret du soir [20 débarrasserait-il enfin de ce côté “papotages" qui le rendait si souvent insupportable?» Télé 59, 11 au 17 janvier 1959, nº742.

34. Martin-Fugier, Anne. 2003. Les Salons de la III République, Art, littérature, politique. Paris: Librairie Académique Perrin.

35. Coté «Rive gauche ", Saint-Germain ou Quartier latin, on trouve par exemple Marie Souris, Barbara, Nicole Louvier, Jean-Roger Caussimon, les Marottes (marionnettes), Félix Marten, Les Trois Ménestrels, Charles Aznavour.

36. La chanteuse a ouvert ce nouveau lieu en 1954, rue Balzac.

37. Frédé ouvre le Carroll's en 1949 au 39 rue de Ponthieu. Dany Dauberson, Annabel ou Colette Mars y chantent. Le lieu est ouvertement « pour les dames ».

38. Hazel Scott, Yvonne Blanc et Art Simmons, Nancy Holloway, Leny Eversong pour le jazz; Mado Robin pour l'opéra. 
39. Madeleine Robinson, Edwige Feuillère, Andrée Debar, Jeanne Fusier-Gir, Marie Bell, Alain Delon... Nous faisons allusion, ici, au cinéma de «qualité française » stigmatisé par François Truffaut (1954).

40. Penet, op. cit, p. 106-107. Voir aussi le duo de Charpini et Brancato [en ligne], consulté le 13 octobre 2016. URL : https://vk.com/video65584699_171555660

41. Cabaret du Soir, RTF, diffusion 28 juin 1958.

42. Strauss, Leo. 2009 [1952, 1989]. La Persécution et l'art d'écrire. Paris : Gallimard, p. 55.

43. Warner, Michael. 2002. Publics and Counterpublics. New York : Zone Books.

44. Wittig, Monique. 2007 [1982, 2001]. « Le point de vue universel ou particulier (avant-note à La Passion de Djuna Barnes)» La Pensée straight. Paris : Éditions Amsterdam, p. 112.

45. Brémond d'Ars, Yvonne (de). 1957. C'est arrivé en plein Paris, passionnante aventure d'antiquaire. Paris : H. Lefebvre.

46. C'est ainsi que Suzy Solidor évoque sa relation formatrice avec l'antiquaire. Latimer, Tirza T. 2005. Women Together/Woman Apart. New Brunswick, New Jersey: Rutgers University Press, p. 108-109.

47. Magazine féminin, RTF, diffusion 25 mai 1958, chronique : Micheline Sandrel ; Journal télévisé Nuit, Vitrines du Faubourg-Saint-Honoré, RTF, diffusion 13 juin 1959, journaliste : Micheline Sandrel (apparition de Mick Micheyl dans ce même sujet) ; L'Art de vivre: Yvonne de Brémond d'Ars, RTF, diffusion 31 janvier 1963, réalisateur : Michel Ayats.

48. Observation récurrente de Catherine Gonnard lorsqu'elle était rédactrice en chef du magazine lesbien mensuel Lesbia Magazine, entre 1986 et 1998.

49. La Garçonne, $2^{\mathrm{e}}$ version (1957), d'après le roman éponyme de Victor Margueritte (1925), réalisé par Jacqueline Audry, avec Andrée Debar, Fernand Gravey, Colette Mars. Dans la première version de Jean de Limur (1936), avec Marie Bell, Arletty, Edith Piaf, une scène se passe à La Vie parisienne, le cabaret de Suzy Solidor. Voir Colette Mars interpréter La Garçonne dans le film de Jacqueline Audry [en ligne], consulté le 13 octobre 2016. URL : https://www.youtube.com/watch? v=wgo89QP7w1M

50. RTF, diffusion 4 février 1957.

51. Cabaret du soir, RTF, diffusion 22 mars 1958.

52. Olivia (1951), réalisatrice: Jacqueline Audry, scénariste: Colette Audry, d'après le roman éponyme de Dorothy Bussy (1949).

53. Nous avons gardé la traduction par Marie Bonaparte et Mme E. Marty (1933) du concept « das Unheimliche », proposé par Freud en 1919.

54. Cabaret du soir, RTF, diffusion 24 janvier 1959.

55. Rochefort, Christiane. 1958. Le Repos du guerrier. Paris : Grasset.

56. Dans Les Aventures du roi Pausole de Pierre Louÿs (1901), chaque nuit, le roi Pausole choisit l'une de ses trois cent soixante-six femmes, dont chacune devient la reine d'un jour pendant une année. Christiane Rochefort s'identifie ainsi à un personnage (très) polygame !

57. Nous appelons ainsi des chansons suffisamment ambiguës pour qu'on ne sache pas si elles sont adressées à un homme ou à une femme, par analogie avec les personnes intersexes, qui revendiquent le droit de ne pas choisir leur sexe, en lien avec les travaux des biologistes comme Anne Fausto-Sterling et Helen Longino.

58. Cabaret du soir, RTF, diffusion 3 mars 1959.

59. L'exposition Made in Algeria: Généalogie d'un territoire (commissaires : Zahia Rahmani \& JeanYves Sarrazin. 2016. Marseille : MUCEM) a récemment montré comment les supports de diffusion véhiculent une vision schématique des colonies qui sert le commerce et le tourisme, masquant la réalité de la vie des populations autochtones, qui sont alors en plein combat pour s'extraire du colonialisme.

60. Sur l'importance des cultures populaires pour la théorie queer, $c f$. De Lauretis, Teresa. 2007. Théorie queer et cultures populaires. De Foucault à Cronenberg. Paris : La Dispute. 
61. Télé Paris à Cannes, RTF, diffusion 9 août 1955.

62. Harvey, John \& Lee, John. 1998 [1996]. Les Hommes en noir, du costume masculin à travers les siècles. Paris : Abbeville Press France.

63. Iconographie [en ligne], consulté le 13 octobre 2016 : http://www.artnet.com/artists/tamarade-lempicka/portrait-de-la-duchesse-de-la-salle-2UpqnRKxR3TLGp-fErG7Bg2.

64. Iconographie [en ligne], consulté le 13 octobre 2016 : http://americanart.si.edu/collections/ search/artist/?id=599.

65. Voir à ce sujet : Latimer. op.cit., p. 51-65.

66. Je vais mourir demain, chanson interprétée par Gribouille dans l'émission Vient de paraître, ORTF, diffusion 25 mai 1965, réalisatrice : Janine Guyon.

67. École des vedettes, RTF, diffusion 19 novembre 1962, réalisateur : Stellio Lorenzi, productrice : Aimée Mortimer, présentateur : Jean-Pierre Aumont [en ligne]. URL : https://www.youtube.com/ watch?v=5N_mnDjS_qc

68. Gribouille est le nom de scène de Marie-France Gaîté. À noter que la scène est presque littéralement retranscrite dans l'ouverture du film de Claude Chabrol, Les Biches (1968), où Why (Jacqueline Sassard), dessinant des biches à la craie sur le Pont-des-Arts, est draguée par Frédérique (Stéphane Audran), qui lui pose les mêmes questions.

69. Orain, Marie-Thérèse. 2001. Gribouille. Je vais mourir demain. Paris : Christian Pirot.

70. Butler, Judith. 1993. "Imitation and Gender insubordination", in The Lesbian and Gay Studies Reader. Abelove, Henri, Aina Barale, Michèle \& Halperin, David M. (dir.). New York/London: Routledge, p. 309.

71. Claudine à l'école (1900), Claudine à Paris (1901), Claudine en ménage (1902), Claudine s'en va (1903), La retraite sentimentale (1907). Série publiée sous le nom de Willy, puis, après 1911, sous la double signature de Colette et Willy jusqu'en 1949, puis sous la signature de la seule Colette, avant que la double signature ne soit rétablie.

72. Louvier, Nicole. 1953. Qui qu'en grogne. Paris : Gallimard.

73. Un rythme à trois temps, qui revient en vedette avec Besame Mucho de Consuelo Velazquez (1940, traduite en 1945).

74. Nicole Louvier chante Nicole Louvier, ORTF, diffusion 28 mai 1964, réalisatrice : Janine Guyon [en ligne]. URL : https://www.youtube.com/watch?v=FWSCvufDoyg

75. Alain, Jeannine, 1958. "Chansons interdites, de Nicole Louvier » Arcadie 54, revue littéraire et scientifique [en ligne], consulté le 14 octobre 2016. URL : http://culture-et-debats.over-blog.com/ article-chansons-interdites-de-nicole-louvier-par-jeannine-allain-116611513.html

76. 36 chansons, RTF, diffusion 2 juin 1957, réalisateur : Lazare Iglesis.

77. Cabaret du soir, RTF, diffusion $1^{\mathrm{er}}$ mars 1958, réalisateur : Jean Kerchbron.

78. Magazine féminin, RTF, diffusion 5 février 1959, productrice: Maïté Célérier de Sannois, journaliste : Micheline Sandrel.

79. Magazine féminin, RTF, diffusion 23 janvier 1965, productrice: Maïté Célérier de Sannois, journaliste : Micheline Sandrel.

80. Preciado, Paul B. (Beatriz). 2011 [2010]. Pornotopie. Playboy et l'invention de la sexualité multimédia. Paris : Flammarion, p. 15.

81. Idem.

82. Cf. note 74 .

83. Faderman, Lilian. 1991. Odd Girls and Twilight Lovers: a History of Lesbian Life in $\mathrm{XX}^{\text {th }} \mathrm{C}$. America. New York: Columbia University Press. Lapovsky Kennedy, Elisabeth \& Davis, Margaret D. 2014 [1993]. Boots of Leather, Slippers of Gold: the History of a Lesbian Community. New York: Routledge. Rupp, Leila J. 1999. A Desired Past. A Short History of Same-Sex Love in America. Chicago: University of Chicago Press. Walker, Lisa. 2001. Looking like What You Are: Sexual Style, Race and Lesbian Identity. New York : New York University Press. 
84. Nous empruntons cette notion $d u$ "plus à l'aise», en anglais "more confortable», à l'anthropologue Gayle Rubin. Rubin, Gayle. 1992. «Of Catamytes and Kings: Reflections on Butch, Gender and Boundaries ", in The Persistent Desire: a Femme/Butch Reader. Nestle, Joan (dir.). Boston : Alyson Publications.

85. Les Joies de la vie: Odette Laure, RTF, diffusion 6 octobre 1958.

86. Cabaret du soir, RTF, diffusion 8 novembre 1958.

87. Yvonne de Brémond d'Ars en serait une autre.

88. De New York à Los Angeles. Ce soir à, RTF, diffusion 3 décembre 1960, réalisateur : Jean-Noël Roy [en ligne], consulté le 13 octobre 2016. URL : https://www.youtube.com/watch?v=DREUM15G-Cs 89. Kosofsky Sedgwick, Eve. 2008 [1990]. Épistémologie du placard. Paris : Amsterdam.

90. Derrida, Jacques \& Stiegler, Bernard. 1996. Échographies de la télévision. Paris : Galilée \& Ina.

91. Joyrich, Lynne. 2001. «Epistemology of the Console " Critical Inquiry [en ligne] 27(3), consulté le 13 octobre 2016. URL: http://www.jstor.org/stable/1344216. Joyrich fait référence au texte d'Eve Kosofsky Sedgwick cité en note 89.

92. Halperin, op.cit., préface à l'édition française, doc. 3, édition Kindle.

93. C'est ce que nous avons pu constater lors de nos trois conférences successives sur le sujet. Gonnard, Catherine \& Lebovici, Elisabeth. "Drôles de Dames ». Séminaire Arts et Féminismes, Fondation Hartung-Bergman à Antibes, 2012; "Elles Sapphichent ", 16 Printemps lesbien de Toulouse, 2013 ; «Elles Sapphichent », 25 Festival Cineffable, 2 novembre 2013. C'est d'ailleurs la raison pour laquelle nous avons décidé de reprendre cette recherche.

94. Halperin, op.cit., chap. 6, doc. 2, édition Kindle.

\section{RÉSUMÉS}

Entre 1955 et 1969, des cultures et sociabilités féminines non normatives apparaissent à la télévision française. Certaines émissions de variétés introduisent des formes de présentation de soi et de relationnalité rompant avec la hiérarchisation binaire des genres. Invisibles aux yeux d'un public non averti, ces figures, qui ne s'identifient pas comme homosexuelles, contribuent cependant à constituer la culture lesbienne francophone de l'après-guerre en objet d'études pour la théorie queer.

Between 1955 and 1969 the French national television has allowed forms of self-presentation and relationnalities, related to non heteronormative cultures and sociabilities. Broadcast regularly, a number of variety shows deployed a range of extraordinary performances. Invisible to an unaware audience, these "pre-Stonewall" figures would never have identified as lesbian, gay or trans. They nevertheless contributed in constituting the francophone post-war lesbian culture as an object of study for queer theory.

\section{INDEX}

Mots-clés : télévision, médias, lesbiennes, sémiologie, homosocialité, stratégies de genre

Thèmes : Recherches

Keywords : television, media, lesbian, semiology, homosociality, gender strategies 


\section{AUTEURS}

\section{CATHERINE GONNARD}

Historienne de l'art, Ina

Catherine Gonnard, ancienne rédactrice en chef de Lesbia Magazine, est documentaliste à l'Ina.

\section{ELISABETH LEBOVICI}

Historienne et critique d'art, AICA

Elisabeth Lebovici, docteure en esthétique et critique d'art, codirige le séminaire « Something You Should Know » à l'EHESS (Paris).

Elles sont ensemble les auteures [2007] de Femmes/artistes, artistes/femmes, Paris de 1881 à nos jours.

Paris : Hazan. 